\title{
Intralesional injection of Candida albicans antigen versus measles, mumps, and rubella vaccine for treatment of plantar warts
}

\author{
Rania Mahmoud Rageh ${ }^{1}$, El-Sayed Shaaban Hewedy ${ }^{1}$, Doaa Salah Hegab ${ }^{1 凶}$ \\ ${ }^{1}$ Department and Venereology Department, Faculty of Medicine, Tanta University, Tanta, Egypt.
}

\begin{abstract}
Introduction: Many therapeutic modalities have been used for management of plantar warts; however, no optimal treatment with high efficacy and no or low recurrence has been explored to date. Intralesional immunotherapy has shown promising results in the treatment of different types of warts. Here we compare the efficacy of Candida albicans-specific antigen versus measles, mumps, and rubella (MMR) vaccine for treatment of plantar warts by intralesional injection.

Methods: Sixty patients with refractory or recurrent plantar warts were randomly divided into two equal groups. Group A was treated with C. albicans antigen and Group B with MMR vaccine. Both groups were injected intralesionally in a single wart every 3 weeks until complete clearance of the wart or for a maximum of five sessions. The patients were followed up for an additional 2 months. Results: $C$. albicans antigen yielded a statistically significant higher cure rate (80.0\%) than MMR vaccine (26.7\%) in the treatment of plantar warts through a mean of 3.98 sessions versus 4.24 sessions, respectively $(p=0.002)$, and both modalities were well tolerated, with no remarkable side effects and no recurrence in cured patients during follow-up.

Conclusions: Intralesional C. albicans antigen injection is an easy and effective treatment tool for plantar warts, even resistant and recalcitrant ones, with no post-procedural downtime and only transient occasional side effects. MMR vaccine is thought to be less effective.
\end{abstract}

Keywords: Candida albicans, immunotherapy, MMR vaccine, plantar warts

Received: 4 August 2020 | Returned for modification: 19 August 2020 | Accepted: 2 September 2020

\section{Introduction}

Plantar warts (verrucae plantaris) are common skin lesions affecting the plantar aspect of the foot and caused by human papillomavirus (HPV) (1).

Given the high propensity for treatment resistance, inconvenience, and recurrence of plantar warts, and due to the absence of a practical and reliable method for prevention, plantar warts remain a therapeutic challenge. Different therapeutic modalities have been used, ranging from topical salicylic acid to tissue ablation, which are frequently associated with pain, scarring, and long recovery time $(2,3)$. Immunotherapy has shown success in treating different types of warts (including recalcitrant, common, anogenital, and plane warts) by combining a targeted approach and upregulation of the host cell-mediated immune response (4-6).

Candida albicans-specific antigen as an injection immunotherapy for common and plantar warts triggers a helpful immune response in most healthy individuals. It is claimed that the antigen propagates a cell-mediated immune response, accompanied by proliferation of peripheral blood mononuclear cells that target the HPV, leading to wart resolution $(7,8)$.

Measles, mumps, and rubella (MMR) vaccine is another modality of wart immunotherapy, especially in recalcitrant ones (9). Intralesional MMR vaccine is thought to be effective in wart treatment through stimulation of a potent non-specific inflammatory response toward HPV-infected cells with subsequent wart resolution through stimulation of the patient's immune system, particularly cell-mediated immunity, to eradicate the virus $(10,11)$.

This study compares the efficacy and safety of $C$. albicans-specific antigen versus MMR vaccine for treatment of plantar warts by intralesional injection.

\section{Materials and methods}

The study included 60 patients with clinically diagnosed plantar warts, who were recruited from the outpatient clinics of the Dermatology and Venereology Department, Tanta University Hospitals, Egypt. Patients over 12 years old with single or multiple plantar warts that were either refractory to treatment (did not show considerable response to at least one destructive therapeutic modality) or recurred at least once after treatment with a tissue-destructive modality were included in the study. The different modalities of treatment available for warts and the relative advantages and drawbacks of immunotherapy, including a possible failure of response to treatment, were clearly declared to the patients. The study protocol was approved by the institutional review board of the ethics committee of the Faculty of Medicine at Tanta University. Patients with acute febrile illness, those that received treatment for their warts during the preceding month of the study, pregnant females, lactating mothers, patients with immunosuppression or other systemic diseases, and those with a history of any hypersensitivity reactions were all excluded. After signing written informed consent, patients were randomly assigned to two equal groups. Patients in Group A were treated with intralesional injection of $C$. albicans antigen (specific hyposensitizing vaccine $C$. albicans antigen, Allergica, concentration 1:100) prepared in hypersensitivity labs at Ain Shams University, Egypt. Patients in Group B were treated with intralesional MMR vaccine (freeze-dried MMR vaccine, $0.5 \mathrm{ml}$ vials, VACSERA, Egypt). In both groups, a dose of $0.3 \mathrm{ml}$ of the immunotherapeutic solution was intralesionally injected into the largest wart at 3-week intervals until complete clearance of the wart or for a maximum of five sessions. Patients that did not 
show any response to the immunotherapeutic regimen after the fifth injection session were offered an alternative treatment option.

\section{Evaluation}

Clinical and photographic evaluation was performed before starting treatment, at every treatment session, and at follow-up after 2 months from the last session by two dermatologists. The treatment response was categorized according to the following score (9): "complete cure" (complete disappearance of the warts and return of normal skin markings) and "no cure" (no response or only reduction in wart size). "No cure" was further divided into "partial response" ( $\geq 50 \%$ decrease in wart size or number but not completely cured) and "no response" $(<50 \%$ decrease in wart size or number).

Adverse effects that occurred during treatment or at follow-up (pain, redness, swelling at the injection site, or systemic manifestations as fever or flu-like symptoms) were reported. significance was set at $p<0.05$

\section{Results}

All the patients included completed the study. Group A included 20 men and 10 women. Their mean age was $31.6+11.3$ years $(S D$; range 13-55). Group B included 24 men and six women. Their mean age was $32.2+11.1$ years $(S D$; range $14-48)$. Most of the patients included in the study ( $80.0 \%$ of the patients in Group A and $73.7 \%$ of those in Group B) had multiple plantar warts.

The demographic data of the patients as well as the site, number, and duration of plantar warts and history of previous treatment are shown in Table 1. Twelve patients in Group A (40.0\%) and eight patients in Group B $(26.7 \%)$ had distant non-plantar, non-genital warts.

In Group A, 24 patients (80.0\%) were completely cured (examples shown in Fig. 1) and six patients (20.0\%) showed a partial response after the five treatment sessions. In Group B, eight patients (26.7\%) were completely cured (Fig. 2), six patients $(20.0 \%)$

Table 1 | Demographic and clinical data of groups studied.

\begin{tabular}{|c|c|c|c|c|}
\hline Variable & $\begin{array}{l}\text { Group A } \\
(n=30)\end{array}$ & $\begin{array}{l}\text { Group B } \\
(n=30)\end{array}$ & Test of significance & $p$-value \\
\hline \multicolumn{5}{|l|}{ Sex } \\
\hline Male, $n(\%)$ & $20(66.7)$ & $24(80.0)$ & $x^{2}=0.68$ & 0.4 \\
\hline Female, $n(\%)$ & $10(33.3)$ & $6(20.0)$ & & \\
\hline \multicolumn{5}{|l|}{ Age (years) } \\
\hline Range & $13-55$ & $14-48$ & $K=0.11$ & 0.73 \\
\hline Mean $\pm S D$ & $31.6+11.3$ & $32.2+11.1$ & & \\
\hline \multicolumn{5}{|l|}{ Duration of plantar warts (months) } \\
\hline Range & $4-18$ & $5-24$ & $U=147$ & 0.16 \\
\hline Mean $\pm S D$ & $6.66 \pm 3.22$ & $10.66 \pm 8.89$ & & \\
\hline \multicolumn{5}{|l|}{ Number of warts, $n(\%)$} \\
\hline Single & $6(20.0)$ & $8(26.7)$ & $x^{2}=0.18$ & 0.66 \\
\hline Multiple & $24(80.0)$ & $22(73.3)$ & & \\
\hline \multicolumn{5}{|l|}{ Previous plantar wart treatment } \\
\hline Surgical treatment & $4(13.3)$ & $1(3.33)$ & $x^{2}=7.04$ & 0.31 \\
\hline Electrocautery & $6(20.0)$ & $8(26.7)$ & & \\
\hline Cryocautery & $16(53.3)$ & $19(63.3)$ & & \\
\hline Topical salicylic acid & $4(13.3)$ & $2(6.7)$ & & \\
\hline Presence of warts in areas other than the sole, $n(\%)$ & $12(40.0)$ & $8(26.7)$ & - & - \\
\hline \multicolumn{5}{|l|}{ Degree of improvement, $n(\%)$} \\
\hline Complete cure & $24(80.0)$ & $8(26.7)$ & $x^{2}=12.62$ & $0.002 *$ \\
\hline Partial response & $6(20.0)$ & $6(20.0)$ & & \\
\hline No response & 0 & $16(53.3)$ & & \\
\hline $\begin{array}{l}\text { Resolution of anatomically distant warts, } \\
n / \text { cases with distant warts (\%) }\end{array}$ & $5 / 12(41.7)$ & $1 / 8(12.5)$ & & \\
\hline \multicolumn{5}{|l|}{ Side effects, $n(\%)$} \\
\hline Mild transient pain on the day of injection & $25(83.3)$ & $5(16.7)$ & $x^{2}=26.13$ & $<0.001^{*}$ \\
\hline Redness & $22(73.3)$ & $7(23.3)$ & $x^{2}=23.3$ & $<0.001^{*}$ \\
\hline Swelling & $25(83.3)$ & $3(10.0)$ & $x^{2}=22.6$ & $<0.001 *$ \\
\hline Flu-like symptoms & $11(36.6)$ & 0 & $x^{2}=26.61$ & $<0.001^{*}$ \\
\hline Ecchymosis & 0 & $2(6.7)$ & $x^{2}=1.03$ & 0.31 \\
\hline
\end{tabular}

$\mathrm{K}=$ Kruskal-Wallis test, $\mathrm{U}=$ Mann-Whitney test, $\mathrm{x}^{2}=$ chi-squared test, ${ }^{*}=$ statistically significant at $p \leq 0.05$.

\section{Statistical analysis}

Data were analyzed using IBM® SPSS statistical software, version 21. Numerical data were presented as means and standard deviations $(S D)$, and categorical data were presented as numbers and percentages. A chi-squared test was used to compare the qualitative data. When the chi-squared test was not appropriate, the Monte Carlo (MC) test was applied. The Mann-Whitney test and Kruskal-Wallis test were used to compare the two means in different groups. Linear correlation analysis was performed using the Spearman coefficient correlation and used for testing the positive or negative associations between different variables. The level of
Table 2 | Number of sessions required for best results and results of follow-up after $\mathbf{2}$ months in totally cured patients in both groups studied.

\begin{tabular}{|c|c|c|c|c|}
\hline & $\begin{array}{c}\text { Total cures, } \\
\text { Group A } \\
(n=24)\end{array}$ & $\begin{array}{c}\text { Total cures, } \\
\text { Group B } \\
(n=8)\end{array}$ & $\begin{array}{c}\text { Test of } \\
\text { significance }\end{array}$ & $p$-value \\
\hline Sessions, $n(\%)$ : & & & $M C=9.32$ & $0.001^{\star}$ \\
\hline Two & $4(16.7)$ & $2(25.0)$ & & \\
\hline Three & $6(25.0)$ & $4(50.0)$ & & \\
\hline Four & $8(33.3)$ & $2(25.0)$ & & \\
\hline Five & $6(25.0)$ & 0 & & \\
\hline \multicolumn{5}{|l|}{ Follow-up, $n(\%)$} \\
\hline Recurrence & 0 & 0 & & \\
\hline No recurrence & $24(100.0)$ & $8(100.0)$ & & \\
\hline
\end{tabular}

$\mathrm{MC}=$ Monte Carlo test for chi-squared, * ${ }^{*}$ statistically significant at $p \leq 0.05$. 
showed a partial response, and 16 patients $(53.3 \%)$ showed no response. The patients in Group A achieved statistically significant better results with a higher incidence of complete cure than those in Group B. The mean number of sessions needed to achieve the best results in patients in both groups as well as their follow-up data are shown in Table 2. The degree of improvement of plantar warts was significantly related to the number of immunotherapeutic injection sessions received by patients in Group A only ( $p=$ 0.037; Fig. 3). It should be noted that $41.7 \%$ of those that had concomitant distant warts among the patients in Group A and 12.5\% of those with distant warts in Group B showed resolution of their distant un-injected warts with immunotherapy (Table 1).

Regarding side effects, there was a statistically significant higher incidence of pain on the day of injection, redness, swell-

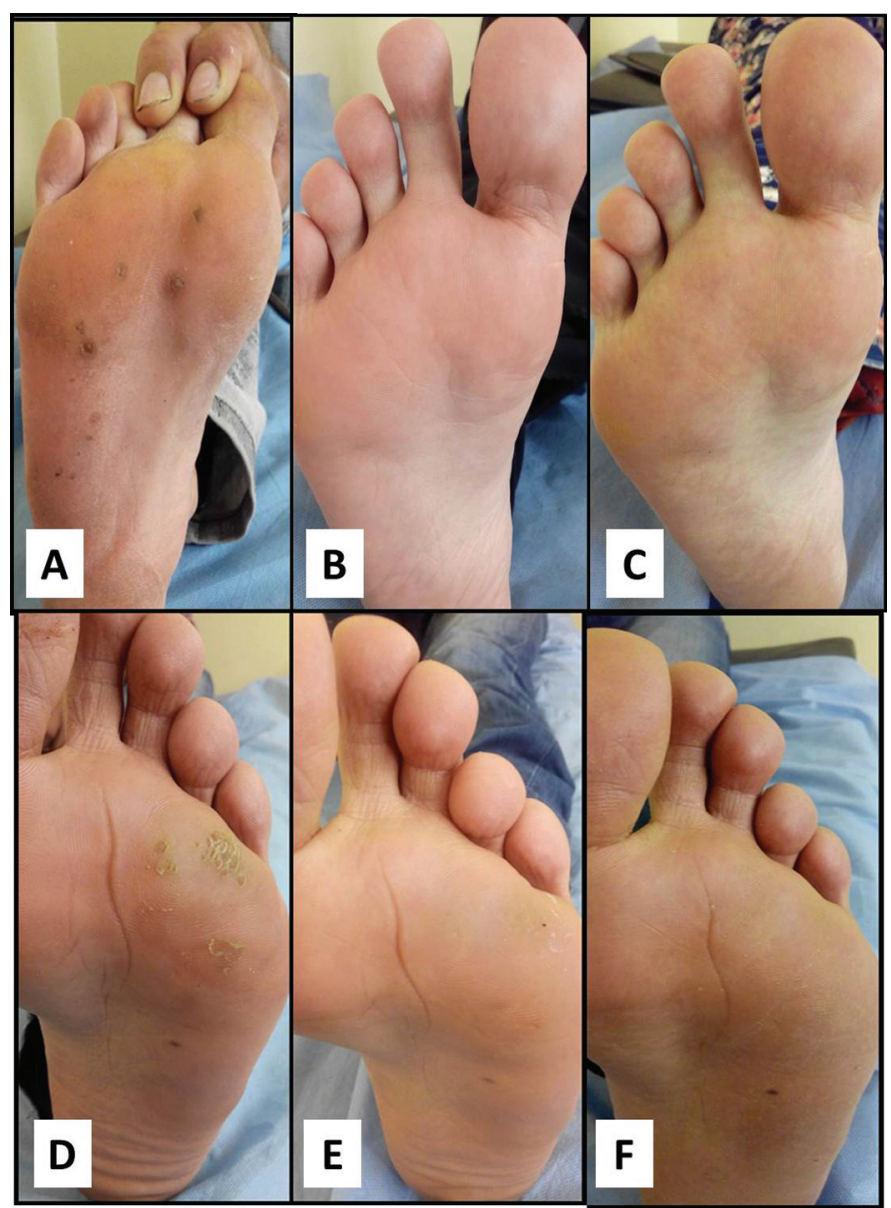

Figure 1 | A 45-year-old male patient with multiple plantar warts A) before treatment, B) after two sessions of $C$. albicans antigen intralesional injection with complete cure, and C) after 2 months of follow-up with no recurrence; and a 30-year-old male patient with multiple plantar warts $D$ ) before treatment, E) after four sessions of $C$. albicans antigen intralesional injection with complete cure, and F) after 2 months of follow-up with no recurrence. ing, and flu-like symptoms in the patients in Group A compared to those in Group B. All these reported side effects were mild, tolerable, and transient (Table 1).

No statistically significant correlations were detected between the degree of improvement of plantar warts and patient age or duration of warts in the two groups studied (Fig. 4).

\section{Discussion}

Intralesional immunotherapy using different skin test antigens and vaccines has shown considerable efficacy and safety in the treatment of different types of warts, even in recurrent and/or resistant lesions $(12,13)$. It could resolve the lesions without any physical injury or scarring, and, in addition, it would augment the host immune response against the causative agent with resolution of distant un-injected lesions (14). To the best of our knowledge, this is the first study comparing the use of $C$. albicans-specific antigen versus the MMR vaccine for treatment of plantar warts by intralesional injections.

In this study, the incidence of complete cure in patients treated with $C$. albicans antigen injection was $80 \%$, which is comparable to several previous studies that utilized the same antigen for wart treatment $(7,8,15,16)$. A literature review shows that several research groups have studied intralesional C. albicans antigen for the treatment of common and genital warts, and that this has shown promising results $(15,17)$. However, to the best of our knowledge, studies regarding its use for plantar warts are relatively few.

In a previous study, Vlahovic et al. used a Luer-lock syringe to inject 0.1 to $0.3 \mathrm{ml}$ of $C$. albicans antigen into either the oldest



Figure 2 | A 20-year-old male patient with multiple plantar warts A) before treatment, B) after five sessions of measles, mumps, and rubella (MMR) vaccine intralesional injection with complete cure, and C) after 2 months of follow-up with no recurrence.

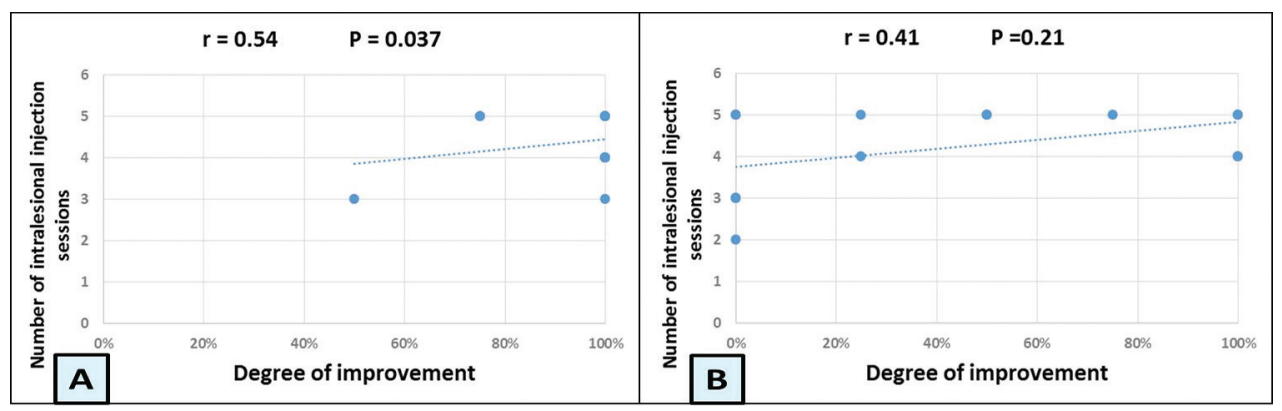

Figure 3 | Correlation between the degree of improvement and the number of intralesional immunotherapy injection sessions $A$ ) in patients treated with $C$. albicans antigen injection with a significant positive correlation and B) in patients treated with measles, mumps, and rubella (MMR) vaccine injection with an insignificant correlation. 


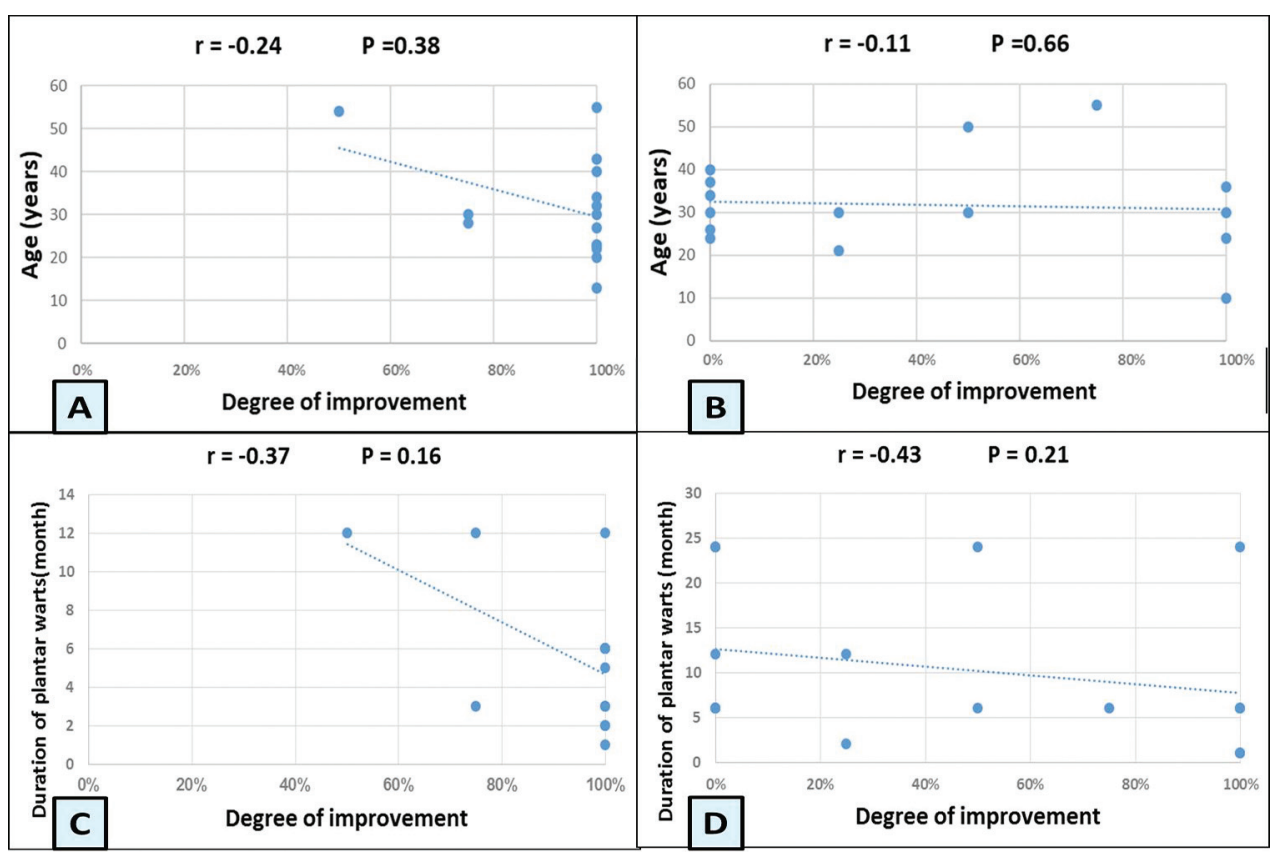

Figure 4 | Correlation between the degree of improvement and patient age with insignificant results A) in patients treated with $C$. albicans antigen injection and B) in patients treated with measles, mumps and rubella (MMR) vaccine injection; and correlation between the degree of improvement and duration of plantar warts (in months) with insignificant results $C$ ) in patients treated with C. albicans antigen injection and D) in patients treated with MMR vaccine injection.

or the largest wart in a sample of 80 patients with plantar warts. They reported total clearance of the lesions in $65 \%$ of their patients, but their study included patients lost to follow-up in the $35 \%$ failure-rate group (7). In fact, the authors of this study believe that doses higher than $0.1 \mathrm{ml}$ yield higher immunogenic potential with preserved safety, whereby $80 \%$ of plantar wart patients achieved a complete cure using $0.3 \mathrm{ml}$ of $C$. albicans antigen and without considerable adverse effects. This dose was fixed and well-tolerated by patients throughout the study. In a similar context, Kim et al. treated 11 patients with non-genital, non-facial warts by injecting $0.3 \mathrm{ml}$ of Candida antigen every 3 weeks for a total of four treatments. Nine patients $(82 \%)$ had complete resolution, and one patient ( $9 \%$ ) achieved a partial response with no increase in adverse effects. These results are in agreement with our results, which strongly advocate the use of higher doses to achieve a greater immunogenic response (17).

In another study, three of seven patients with HIV that were injected intralesionally with Candida antigen (0.3 ml every 3 weeks) achieved complete clearance of their common warts (18). The immunocompromised state of HIV patients and the crucial role of cell-mediated immunity in achieving response with $C$. albicans antigen immunotherapy might explain their lower cure rates (42.8\%), which are still considered promising for this population of patients.

It should be noted that no recurrence was detected in completely cured cases of Group A in this study on follow-up, and this was in agreement with the studies by Vlahovic et al., Majid et al., and Nofal et al. $(7,8,16)$. The flared body immune response against HPV might explain the persistent incapability of the virus and its inoculated nearby particles to induce recurrence in cured patients.

Regarding side effects of $C$. albicans immunotherapy in patients in Group A, transient tolerable side effects such as flu-like symptoms, pain during and after injection, and redness and swelling at the injection site were reported in this study and were similar to those reported in several previous studies $(8,16-19)$. In fact, the authors of this study believe that the occurrence of flu- like symptoms and local reactions at the injected wart are good signs, indicating that a major immune reaction will resolve the wart and destroy the HPV (in a manner that is quite similar to the immunizing pre-test of intralesional antigen injection adopted by several research groups to predict the response to treatment). We noticed that the patients that experienced the most severe adverse effects were those that showed the best curative results for their warts.

The degree of improvement of plantar warts in patients in Group A was significantly related to the number of sessions received, and this was in agreement with several previous studies (15-17).

Although MMR vaccine has shown promising results in previous reports when used in common, multiple, and recalcitrant cutaneous warts $(11,20-24)$, data on its efficacy in plantar warts are relatively sparse in published literature.

Regarding the degree of improvement in patients in Group $\mathrm{B}$, the incidence of complete response in this study $(26.7 \%)$ was lower than that found in the studies by Nofal et al. (63\%), Zamanian et al. (75\%), and Awal et al. (68\%) (9, 20, 24). This might be attributed to variation in the type of warts treated, whereby plantar warts are thought to be more resistant to immunotherapy than other types of common extragenital warts. Moreover, Zamanian et al. used a higher dose of MMR injection $(0.5 \mathrm{ml})$ than our study (0.3 ml) (20).

In a recent study, Abd El-Magiud et al. tested MMR vaccine versus cryotherapy for treating common and plantar warts, and they achieved a 70\% cure in the MMR group with a $0.5 \mathrm{ml}$ intralesional vaccine injection, biweekly for only three sessions (25). It should be taken into consideration that their patients in the MMR group included patients with common warts in addition to those with plantar ones ( $50 \%$ for each), whereby common warts are thought to be more readily responsive to therapy. In addition, they achieved their results by injecting higher volume $(0.5 \mathrm{ml})$.

It is noteworthy that none of the cured patients in Group B in this study developed recurrence after 2 months of follow-up, and this was in agreement with Rezai et al.'s study (11), whereas in 
studies by Na et al. (21) and Nofal et al. (9) recurrence developed in $5.6 \%$ and $4.8 \%$, respectively, of those that had a complete response at the 6-month follow-up visit. The shorter period of follow-up in this study might explain this variation in the results of recurrence.

Regarding side effects in Group B, this study showed that pain on the day of injection occurred in only $16.7 \%$ of patients in Group B. Their pain was tolerable and did not affect the compliance of patients to continue the treatment protocol; this was in agreement with several previous studies $(9,11,23,25)$. In our study, flu-like symptoms were not reported in Group B, and this was in agreement with the findings by $\mathrm{Na}$ et al. (21). In contrast, Nofal et al. (9), Zamanian et al. (20), and Abd El-Magiud et al. (25) reported flu-like symptoms in $12.3 \%, 30 \%$, and $15 \%$ of their patients, respectively.

Collectively speaking, adverse effects to immunotherapy have been stated to be mild and generally insignificant, in addition to the absent or low recurrence rates that encourage its use in clinical practice $(26,27)$.

It should be mentioned that patients treated with $C$. albicans antigen immunotherapy achieved significantly better results, with a higher incidence of complete cure (80.0\% versus $26.7 \%$, $p=0.002$ ), and a smaller number of injection sessions required than for those treated with MMR vaccine. The variable immune response of immunocompetent individuals to different types of immunotherapeutic modalities is a common clinical observation that requires future research for explanation. Therefore, shifting from one type of immunotherapy to another might be a helpful option for patients that failed to develop a response to a certain immunotherapeutic agent.

There were some limitations to this study. The small number of the patients studied, the absence of a control group, and the short follow-up period are the main limitations. In addition, a pre-test with intralesional injection of a test dose of the immunotherapeutic drug ( $0.1 \mathrm{ml})$ to rule out non-responders as well as hypersensitive patients was not performed.

\section{Conclusions}

This study indicates that intralesional injection of either C. albicans antigen or MMR vaccine may have some potential advantages in treating patients with plantar warts because they are well tolerated, have a low cost, and result in no scarring or post-injection downtime in addition to the single injection site. C. albicans antigen immunotherapy yielded better results with a significantly higher incidence of a complete cure in resistant and recurrent plantar warts (regardless of the number of lesions) than intralesional MMR vaccine, and this was achieved with significantly fewer treatment sessions. A major additional advantage of this modality over most traditional treatments is its effectiveness in multiple as well as distant un-injected lesions and a lower risk of future recurrence in cured cases. Our study recommends intralesional $C$. albicans antigen injection as a helpful addition to the current therapeutic armamentarium against plantar warts, especially in multiple, resistant, and recurrent cases.

\section{References}

1. Witchey DJ, Witchey NB, Roth-Kauffman MM, Kauffman MK. Plantar warts: epidemiology, pathophysiology, and clinical management. J Am Osteopath Assoc. 2018;118:92-105.

2. Rivera A, Tyirng SK. Therapy of cutaneous human papillomavirus infections. Dermatol Ther. 2004;17:4411-8.

3. Vlahovic TC, Khan MT. The human papillomavirus and its role in plantar warts: a comprehensive review of diagnosis and management. Clin Podiatr Med Surg. 2016;33:337-53.

4. Aldahan AS, Mlacker S, Shah VV, Kamath P, Alsaidan M, Samarkandy S, et al. Efficacy of intralesional immunotherapy for the treatment of warts: a review of the literature. Dermatol Ther. 2016;29:197-207.

5. Johnson S, Horn T. Intralesional immunotherapy for warts using a combination of skin test antigens: a safe and effective therapy. J Drugs Dermatol. 2004;3:263.

6. Fields JR, Saikaly SK, Schoch JJ. Intralesional immunotherapy for pediatric warts: a review. Pediatr Dermatol. 2020;37:265-71.

7. Vlahovic TC, Spadone S, Dunn SP, Fussell T, Hersh I, Green T, et al. Candida albicans immunotherapy for verruca plantaris. J Am Podiatr Med Assoc. 2015;105: 395-400.

8. Majid I, Imran S. Immunotherapy with intralesional Candida albicans antigen in resistant or recurrent warts: a study. Indian J Dermatol. 2013;58:360.

9. Nofal A, Nofal E, Yosef A, Nofal H. Treatment of recalcitrant warts with intralesional measles, mumps and rubella vaccine: a promising approach. Int J Dermatol. 2015;54:667-71.

10. Horn TD, Johnson SM, Helm RM, Roberson PK. Intralesional immunotherapy of warts with mumps, Candida, and Trichophyton skin test antigens. Arch Dermatol. 2005;141:589-94.

11. Rezai MS, Ghasempouri H, Asqary Marzidareh O, Yazdani Cherati J, Rahmatpour Rokni G. Intralesional injection of the measles-mumps-rubella vaccine into resistant palmoplantar warts: a randomized controlled trial. Iran J Med Sci. 2019; 44:10-7.

12. Gupta S, Malhotra AK, Verma KK, Sharma VK. Intralesional immunotherapy with killed Mycobacterium w vaccine for the treatment of anogenital warts: an open label pilot study. J Eur Acad Dermatol Venereol. 2008;22:1089-93.

13. Wananukul S, Chatproedprai S, Kittiratsacha P. Intralesional immunotherapy using tuberculin PPD in the treatment of palmoplantar and periungual warts. Asian Biomedicine. 2009;3:739-43.

14. Sinha S, Relhan V, Garg VK. Immunomodulators in warts unexplored or ineffective? Indian J Dermatol. 2015;60:118-29.
15. Elmaadawy EH, Shams SS, Hegab DS, Zaki RA. Pulsed-dye laser versus intralesional Candida albicans antigen injection in treatment of genital warts. Acta Dermatovenerol Alp Pannonica Adriat. 2019;28:21-6.

16. Nofal A, Marei A, Amer A, Amen H. Significance of interferon gamma in the pre diction of successful therapy of common warts by intralesional injection of Candida antigen. Int J Dermatol. 2017;56:1003-9.

17. Kim KH, Horn TD, Pharis J, Kincannon J, Jones R, O’Bryan K, et al. Phase 1 clinical trial of intralesional injection of Candida antigen for the treatment of warts. Arch Dermatol. 2010;146:12.

18. Wong A, Crawford RI. Intralesional Candida antigen for common warts in people with HIV. J Cutan Med Surg. 2013;17:313-5.

19. Summers P, Richards-Altmon P, Halder R. Treatment of recalcitrant verruca vulgaris with Candida antigen in patient with human immunodeficiency virus. J Drugs Dermatol. 2009;8:268-9.

20. Zamanian A, Mobasher P, Jazi GA. Efficacy of intralesional injection of mumpsmeasles-rubella vaccine in patients with wart. Adv Biomed Res. 2014;3:107.

21. $\mathrm{Na} \mathrm{CH}$, Choi H, Song SH, Kim MS, Shin BS. Two-years experience of using the measles, mumps and rubella vaccine as intralesional immunotherapy for warts. Clin Exp Dermatol. 2014;39:583-9.

22. Saini S, Dogra N, Dogra D. A prospective randomized open label comparative study of efficacy and safety of intralesional measles, mumps, rubella vaccine versus $100 \%$ trichloroacetic acid application in the treatment of common warts. Int J Res Med Sci. 2016;4:1529-33.

23. Meena D, Sharma S, Chauhan P. Measles, mumps and rubella vaccine in genital warts: a case report of two patients. Indian J Sex Transm Dis AIDS. 2018;39:133-

24. Awal G, Kaur S. Therapeutic outcome of intralesional immunotherapy in cutaneous warts using the mumps, measles and rubella vaccine. J Clin Aesthet Dermatol. 2018;11:15-20.

25. Abd El-Magiud EM, Abd El-Samea GM, Gaber HD. Intralesional injection of measles, mumps, and rubella vaccine versus cryotherapy in treatment of warts: a randomized controlled trial. Dermatol Ther. 2020;33:e13257.

26. Nofal A, Salah E, Nofal E, Yosef A. Intralesional antigen immunotherapy for the treatment of warts: current concepts and future prospects. Am J Clin Dermatol. 2013;14:253-60.

27. Nofal A, Albalat W, Ismail A, Khattab FM. Immunotherapeutic modalities for the treatment of recalcitrant plantar warts: a comparative study. J Dermatolog Treat. $2020 ; 1-6$. 